\section{Valores de magnésio no humor vítreo de bovinos abatidos para consumo no sul do Estado do Rio de Janeiro}

\author{
Values of magnesium in the vitreous humor of bovine in the \\ south of the state of Rio de Janeiro
}

\author{
Fátima Itzel Valdés Peréz' ${ }^{1}$ Rita de Cassia Campbell Machado Botteon²*, Bianca Pachiel Medeiros ${ }^{3}$; \\ Jéssica D'Avilla de Assis ${ }^{4} \&$ Paulo de Tarso Landgraf Botteon ${ }^{2}$ \\ 'Medica veterinária, Mestranda. Programa de Pós-graduação em Medicina Veterinária - PPGMV, Universidade Federal Rural do \\ Rio de Janeiro - UFRRJ, Seropédica, RJ, Brasil \\ ${ }^{2}$ Médicos veterinários. Departamento de Medicina e Cirurgia Veterinária, Instituto de Veterinária, Universidade Federal Rural do \\ Rio de Janeiro - UFRRJ, Seropédica, RJ, Brasil \\ ${ }^{3}$ Médica veterinária, Residente Hospital Veterinário de Grandes Animais. Universidade Federal Rural do Rio de Janeiro - UFRRJ, \\ Seropédica, RJ, Brasil \\ ${ }^{4}$ Graduanda em Medicina Veterinária. Universidade Federal Rural do Rio de Janeiro - UFRRJ, Seropédica, RJ, Brasil
}

\section{Resumo}

Devido à incidência elevada de hipomagnesemia em alguns países e inexistência de levantamentos realizados no Brasil, objetivou-se avaliar as concentrações de magnésio em humor vítreo de bovinos abatidos no Rio de Janeiro, no início da primavera, visando investigar a ocorrência de animais em condições de desenvolver a hipomagnesemia clínica. Foram avaliados olhos de 116 bovinos adultos. Os olhos, um de cada animal, foram obtidos imediatamente após a morte e manipulados para obtenção do humor vítreo, em ambiente refrigerado. O vítreo foi colhido por aspiração com agulha calibre 27x7 mm, inserida no canto lateral do olho. Aproximadamente 1,0 ml do líquido foi centrifugado a 3000g, por 15 minutos. O sobrenadante foi aspirado com pipeta descartável, acondicionado em frascos sem conservantes e mantido a $-20{ }^{\circ} \mathrm{C}$. Foram realizados ensaios para magnésio utilizando-se kits comerciais em espectrofotômetro

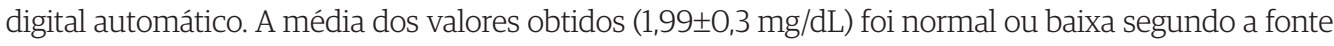
consultada. No humor vítreo de 66 animais (56,9\%) a concentração do magnésio foi menor que 2,02 mg/dL, indicativo de hipomagnesemia, configurando uma condição de alta probabilidade de a hipomagnesemia clínica (tetania) ocorrer nas condições do presente estudo. A possibilidade de realizar o diagnóstico post-mortem por meio da análise do humor vítreo é importante para a adoção de medidas preventivas e/ou curativas em rebanhos em condições de risco.

Palavras-chave: hipomagnesemia, tetania, lactação, pastagens, olhos, doenças metabólicas.

\begin{abstract}
Due to the high incidence of hypomagnesemia in some countries and the absence of studies in Brazil, we want to evaluate the concentrations of magnesium in the vitreous humor of slaughtered bovines in the state of Rio de Janeiro in early spring, aiming to investigate the occurrence of animals under conditions of clinical hypomagnesemia. A total of 116 eyes of adult bovines of different ages and breeds were evaluated. We extracted an eye of each animal to evaluate, immediately after the slaughter and were manipulated to obtain the vitreous humor, under appropriate temperatures conditions. The vitreous humor was collected by aspiration with a $27 \times 7 \mathrm{~mm}$ needle, inserted in the lateral corner of the eye. Approximately $1.0 \mathrm{ml}$ of liquid was centrifuged (3000rpm, 15 minutes), the supernatant aspirated with a disposable micropipette, packaged in eppendorfs tubs without preservatives was kept at $-20^{\circ} \mathrm{C}$. Tests were performed to determine magnesium concentrations using commercial kits in spectrophotometer automatic digital. The mean of the values obtained $(1.99 \pm 0.3 \mathrm{mg} / \mathrm{dL})$ was normal or low according to the source consulted. In 66 animals $(56,9 \%)$ the concentration of magnesium in the vitreous humor was lower, $2.02 \mathrm{mg} / \mathrm{dL}$, indicative of hypomagnesemia, demonstrating a high probability condition of clinical hypomagnesemia (tetany) can develop in the conditions outlined in the present study. The possibility of making a post-mortem diagnosis through vitreous humor analysis is important for the prevention of these diseases and to search for curative measures in those risky areas.
\end{abstract}

Keywords: hypomagnesemia, tetany, lactation, pastures, eyes, metabolic diseases. \section{BJ $\mathbb{M}$ Brazilian Journal of Veterinary Medicine \\ p-ISSN 0100-2430 e-ISSN 2527-2179

Como citar: Peréz, F. I. V., Botteon, R. C. C. M., Medeiros, B. P., Assis, J. D., \&Botteon, P. T. L. (2017). Valores de magnésio no humor vítreo de bovinos abatidos para consumo no sul do Estado do Rio de Janeiro. Brazilian Journal of Veterinary Medicine, 39(4), 264-269. doi: 10.29374/2527-2179. bjvm009917

Fonte de financiamento: JDA é Bolsista PIBIC/CNPq.

Conflito de interesses: Os autores declaram não haver conflito de interesses que precisam ser informados

Recebido: Dezembro, 03, 2017

Aceito: Fevereiro, 28, 2018.

O estudo foi realizado na Universidade Federa Rural do Rio de Janeiro - UFRRJ, Seropédica, RJ, Brasil.

\section{*Correspondência}

Rita de Cassia Campbell Machado Botteon Departamento de Medicina e Cirurgia Veterinária, Instituto de Veterinária, Universidade Federal Rural do Rio de Janeiro - UFRRJ

Rodovia BR 465, Km 07,

CEP 23890-000 - Seropédica (RJ), Brasil

E-mail: ritabotteon@gmail.br
Copyright Peréz et al. Este é um artigo publicado em acesso aberto (Open Access) sob a licença Creative Commons Attribution Non-Commercial, que permite uso, distribuição e reprodução em qualquer meio, sem restrições desde que sem fins comerciais e que o trabalho original seja corretamente citado. 


\section{Introdução}

Em bovinos, o diagnóstico post-mortem de várias enfermidades, especialmente as que cursam com morte súbita, é difícil ou impossível de realizar mediante necropsia devido à falta de lesões específicas ou ao avançado estado de autólise. Também é improvável a obtenção de sangue em condições de análise devido às rápida degradação e contaminação microbiana dos tecidos e fluidos corporais (Cseh, 1983). Seu curso agudo impede, em muitos casos, observar sinais clínicos, e frequentemente os animais são encontrados mortos. Neste contexto, a análise do humor vítreo é útil, sobretudo para o diagnóstico de hipomagnesemia, uma doença metabólica que normalmente ocorre como surtos (Ramírez et al., 1998), em condições específicas (Zelal, 2017) e causa a morte de até 20\% dos animais afetados (McDowell, 1992). Em vacas leiteiras a incidência é variável sendo mais importante a forma subclínica crônica por predispor à hipocalcemia (Corbellini, 1998). O humor vítreo que é fácil de obter e analisar permite corroborar a suspeita clínica de forma rápida e econômica.

O vítreo é relativamente bem protegido da contaminação microbiana e tem a vantagem de manter a concentração de magnésio estável por 48 horas, em temperatura de até $23^{\circ} \mathrm{C}$ (Lincoln \& Lane, 1985). Por estas razões pode ser utilizado quando o sangue não é uma amostra viável (Kalra et al., 2016).

O vítreo tem sido muito utilizado em patologia forense. Atualmente as possibilidades investigativas são inúmeras e incluem DNA (Belsey \& Flanagan, 2016), anticorpos (Tormey, 2016), choque anafilático (Han et al., 2015), glicemia (Boulagnon et al., 2011), gestação (Han et al., 2015), álcool e drogas (Metushi et al., 2016). Em veterinária, a análise do vítreo é pouco empregada, havendo relatos de sua aplicação diagnóstica na hipomagnesemia (McDowell 1992; Ramírez et al., 1998; Mattioli et al., 2000; McCoy et al., 2001), alterações renais (Choo-Kang et al., 1983) e intoxicações (Lincoln \& Lane, 1985).

No Brasil, há poucas informações sobre a incidência de doenças metabólicas (incluindo a hipomagnesemia), bem como sobre suas implicações. Devido à incidência elevada em alguns países, em condições específicas, e inexistência de levantamentos realizados no Brasil, objetivou-se avaliar as concentrações de magnésio em humor vítreo de bovinos abatidos no Rio de Janeiro, no início da primavera, visando investigar a ocorrência de animais em condições de desenvolver a hipomagnesemia clínica.

\section{Materiais e métodos}

Foram avaliados olhos de 116 bovinos adultos, de diferentesidades e raças, abatidos para consumo segundo normas do Ministério da Agricultura Pecuária e Abastecimento, no Frigorífico Uirapuru, em Barra Mansa - RJ. As coletas foram autorizadas pela Defesa Agropecuária e Coordenadoria de Controle de Qualidade de Produtos Agropecuários Industrializados do Rio de Janeiro.

Os olhos, um de cada animal, foram obtidos em novembro de 2017, imediatamente após a morte, e a enucleação praticada na sala de abate. Em seguida foram acondicionados em sacos plásticos e transportados em caixas isotérmicas contendo gelo até o Laboratório de Pesquisas Clínicas, no Instituto de Veterinária da Universidade Federal Rural do Rio de Janeiro onde foram lavados em água corrente e manipulados para obtenção do humor vítreo, em ambiente refrigerado, imediatamente após o transporte.

O humor vítreo foi colhido por aspiração com seringa descartável de 5,0 mL e agulha hipodérmica calibre $27 \times 7 \mathrm{~mm}$, inserida no canto lateral do olho. Aproximadamente 1,0 ml do líquido foi transferido para um frasco estéril e centrifugado a 3000g por 15 minutos (Grabherr et al., 2012). O sobrenadante foi aspirado com pipeta descartável, acondicionado em frascos sem conservantes e mantido a $-20{ }^{\circ} \mathrm{C}$ até o momento das análises. Foram realizados ensaios bioquímicos para magnésio utilizando-se kits comerciais (Biosystems) em espectrofotômetro digital automático (Biosystems A15).

Os resultados foram apresentados como média e desvio padrão e discutidos com base nos dados da literatura especializada. 


\section{Resultados e discussão}

A concentração normal de magnésio no humor vítreo de bovinos saudáveis a 230C por até 48 horas após a morte varia de 1,8 a 2,7 mg/dL (Corbellini et al., 1992; Lincoln \& Lane, 1985) ou entre 1,86 e 2,28 mg/dL (Wittwer et al., 1992). Os valores aqui obtidos (Tabela 1) variaram entre 1,28 e 3,35 mg/dL, com concentração mínima menor e máxima maior que a descrita por esses

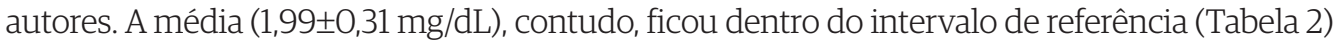
descrito por Lincoln \& Lane (1985), Corbellini et al. (1992) e Wittwer et al. (1992).

Matioli et al. (2000) ao avaliarem amostras de sangue e humor vítreo de bovinos com suspeita de hipomagnesemia, utilizaram o Mg no humor vítreo Humor abaixo de $2.02 \mathrm{mg} / \mathrm{dL}$ como um indicador do nível plasmático ante mortem correspondente a hipomagnesemia (abaixo de 1,80 mg/dL). As concentrações de magnésio variaram entre 1,11 e1,90 mg/dL (2,67 a 4,62 mmol/L), sendo mais baixas nos animais com sinais clínicos, chegando ao extremo de 0,24 mg/dL (0,098 mmol/L).

No presente estudo, em que se avaliou a concentração do magnésio no humor vítreo de 116 bovinos adultos sem histórico de hipomagnesemia, 66 amostras (56,9\%) tiveram a concentração de magnésio menor que 2,02 mg/dL, que conforme Matioli et al. (2000) é um indicador de hipomagnesemia ante mortem (plasma $<1,80 \mathrm{mg} / \mathrm{dL}$ ). Em nenhuma amostra se obteve concentração menor que 1,1 mg/dL sugerida por Corbellini et al. (1992) como indicativa de hipomagnesemia, e em apenas uma a concentração do magnésio foi menor que 1,34 mg/dL indicada por McCoy (2004) como hipomagnesemia. No entanto, obteve-se 30 amostras (25,9\%) com magnésio no humor vítreo menor que 1,80 mg/dL, que corresponde ao limite mínimo do intervalo de referência descrito por Lincoln \& Lane (1985), Corbellini et al. (1992) e Wittwer et al. (1992).

Lincoln \& Lane (1985), McCoy et al. (2001), McLaughlin \& McLaughlin (1987), Ramírez et al. (1998) e Wittwer et al. (1992) encontraram altas correlações entre a concentração de magnésio no humor vítreo post mortem e no sangue antes da morte, e consideraram que o humor vítreo é adequado para avaliação da hipomagnesemia. Por outro lado, Hanna et al. (1990), McCoy \& Kennedy (1994) e Matioli et al. (2000) obtiveram correlações baixas e consideraram seu uso questionável. Ainda que não haja consenso entre os autores, as resultados indicam que o humor vítreo pode ser útil para detectar desequilíbrios de $\mathrm{Mg}^{2+}$ durante pelo menos 48 horas após a morte, desde que a temperatura ambiental pós-morte não ultrapasse $23^{\circ} \mathrm{C}$. Foram detectados valores significativamente mais altos de magnésio no vítreo que no plasma (Ramírez et al.,1998; Wittwer et al., 1992), e dessa forma, considera-se que valores baixos no vítreo são adequados

Tabela 1. concentração de magnésio e cálcio (mg/dL e mmol/L) em humor vítreo de bovinos adultos abatidos na região sul do Estado do Rio de Janeiro, na primavera e inicio das chuvas (novembro de 2017).

\begin{tabular}{lcc}
\hline & $\mathrm{mg} / \mathbf{d L}$ & $\mathrm{mmol} / \mathbf{L}$ \\
\hline Média & 1,99 & 0,90 \\
Desvio padrão & 0,31 & 0,14 \\
Mínimo & 1,28 & 0,58 \\
Máximo & 3,35 & 1,51 \\
\hline
\end{tabular}

Tabela 2. Valores de referência para o magnésio em humor vítreo de bovinos sadios, com suspeita de hipomagnesemia e com hipomagnesemia e tetania.

\begin{tabular}{ccc}
\hline Autor(es)/ano & mg/dL & Condição \\
\hline Lincoln \& Lane (1985) & 1,8 a 2,76 & Sadios \\
Corbellini et al. (1992) & 1,8 a 2,7 & Sadios \\
Wittwer et al. (1992) & 1,86 a 2,28 & Sadios \\
Matioli et al. (2000) & 2,67 a 4,62 & Suspeita de hipomagnesemia \\
McCoy (2004) & $<1,34$ & Com hipomagnesemia e tetania \\
\hline
\end{tabular}


para prever a hipomagnesemia. Os valores aqui obtidos são relevantes sob o ponto de vista da possibilidade de ocorrência da tetania hipomagnesêmica nas condições deste estudo.

A incidência de hipomagnesemia conhecida como tetania das pastagens ou do pasto, tetania da lactação ou tetania dos transportes é variável, e em algumas regiões pode ocorrer na forma de surtos, com grande número de animais acometidos e mortalidade de até15\% (Wittwer et al.,1987). Embora seja possível localizar um artigo referente à frequência de alterações no metabolismo de cálcio e magnésio em equinos com disfunção gastrointestinal (Coelho et al., 2012), no Brasil, não há relatos de incidência e mortalidade de bovinos por hipomagnesemia e não há estimativas oficiais de sua ocorrência. Destaca-se a possibilidade de subnotificação e falhas de diagnóstico em função do desconhecimento da possibilidade de sua ocorrência.

A causa exata da tetania hipomagnesêmica em ruminantes ainda não foi definida. O fator primário parece ser a inadequada absorção de magnésio no trato digestivo, sob a influência de fatores individuais, ambientais e dietéticos (Weiss, 2004). A deficiência na ingestão de magnésio e mais recentemente a alta ingestão de K e Na podem ser importantes na etiologia da hipomagnesemia (Zelal, 2017). No Brasil, uma grande parcela dos animais de produção consomem dietas que não suprem as suas exigências em relação aos minerais. Os alimentos mais comumente utilizados contêm proporções desequilibradas, deficientes ou com excesso de minerais, provocando sérios distúrbios metabólicos (Mendonça Junior et al., 2011). O predomínio de condições carenciais marginais, caracterizadas por manifestações clínicas pouco expressivas e inespecíficas, torna difícil o diagnóstico clinico e de acordo com Kaneko et al. (1997) e Balarin et al. (1998), a avaliação dos sintomas possui importância limitada. Quanto à hipomagnesemia, um transtorno metabólico-nutricional, as condições em que a doença ocorre com maior frequência, como pastos de brotação exuberante no inicio da primavera e mudança brusca de alimentação, transporte e estresse, são provavelmente tão comuns aos rebanhos brasileiros quanto em países em que a doença apresenta alta prevalência descrita. Desta forma, a identificação de animais com hipomagnesemia nas condições de criação brasileira é relevante para destacar a possibilidade de ocorrência da doença. A possibilidade de realizar o diagnóstico post mortem por meio da análise do humor vítreo é fundamental para a adoção de medidas preventivas e/ou curativas em rebanhos em condições de risco.

Matioli et al. (2000) destacaram que as discrepâncias observadas em relação à aplicação do HV como amostra biológica para diagnóstico de hipomagnesemia pode dever-se ao fato de a maioria dos estudos terem sido desenvolvidos com animais sadios, abatidos para consumo, sem que as condições para desenvolvimento da hipomagnesemia sejam observadas. Neste contexto, no presente estudo foram amostrados animais abatidos para consumo, na primavera, tendo-se definido o inicio do período chuvoso, após uma semana de alta precipitação pluviométrica para coletas, considerando as condições da pastagem em brotação e o transporte dos animais como fatores predisponentes da tetania hipomagnesêmica (Littledike et al., 1981; Beede et al., 1992; Goff, 1998).

Os resultados obtidos demonstraram em concordância com observações de Edwards et al. (2009) que a aferição de magnésio nos fluidos oculares de ruminantes após a morte é útil e de fácil aplicação, podendo ser um excelente método auxiliar ao veterinário de campo na elucidação da causa de mortes súbitas em bovinos, uma condição comum no Brasil e frequentemente relacionada a intoxicação por plantas, intoxicação por organofosforados, carbúnculo hemático, hemoglobinúria bacilar e fulguração (Estima-Silva et al., 2016). Na maioria dos casos, o diagnóstico definitivo não é estabelecido e a avaliação do magnésio no humor vítreo de animais mortos pode ser valiosa.

Conclui-se que a hipomanesemia ocorre em rebanhos do Sul do Estado do Rio de Janeiro em períodos de chuvas e brotação de pastagens, sendo o transporte, o estresse e a restrição alimentar i, fatores importantes para sua ocorrência. A forma clinica, caracterizada por tetania e morte súbita é provavelmente não diagnosticada e notificada.

\section{Referências}

Balarin, M. R. S., Lisbôa, J. A. N., Kohayagawa, A., \& Kuchembuck, A. R. G. (1998). Efeito da nutrição mineral sobre as excreções fracionadas urinárias de cálcio e de fósforo em novilhos da raça Nelore. Semina: Ciências Agrárias, 19(1), 7-12. http://dx.doi.org/10.5433/1679-0359.1998v19n1p7. 
Beede, D. K., Sánchez, W. K., \& Wang, C. (1992). Macrominerals. In H. H. Van Horn \& C. J. Wilcox (Eds.), Large dairy herd management (pp. 272-286). Champaign: American Dairy Science Association.

Belsey, S. L., \& Flanagan, R. J. (2016). Postmortem biochemistry: Current applications. Journal of Forensic and Legal Medicine, 41, 49-57. http://dx.doi.org/10.1016/j.jflm.2016.04.011. PMid:27131037.

Boulagnon, C., Garnotel, R., Fornes, P., \& Gillery, P. (2011). Post-mortem biochemistry of vitreous humor and glucose metabolism: an update. Clinical Chemistry and Laboratory Medicine, 49(8), 1265-1270. http://dx.doi. org/10.1515/CCLM.2011.638. PMid:21663468.

Choo-Kang, E., McKoy, C., \& Escoffery, C. (1983). Vitreous humor analytes in assessing the postmortem interval and the antemortem clinical status. The West Indian Medical Journal, 32(1), 23-26. PMid:6868567.

Coelho, C. S., Fonseca, L. A., \& Souza, V. R. C. (2012). Prevalência de hipocalcemia e hipomagnesemia em equinos com distúrbios gastrintestinais. Arquivo Brasileiro de Medicina Veterinária e Zootecnia, 64(3), 755-758. http:/l dx.doi.org/10.1590/S0102-09352012000300030.

Corbellini, C. N., Mangoni, A., Patalogoity, J. F., \& Cerutti de Mattos, A. A. (1992). Tetania hipomagnesémica em bovinos (pp. 1-8, Bolm Inform., Serie Técnica, 23). Argentina: Proyecto Ganadero E.E.A. INTA Pergamino.

Corbellini, N. C. 1998. Etiopatogenia e controle da hipocalcemia e hipomagnesemia em vacas leiteiras. In F. D. H. González, H. P. Ospina, \&, J. O. J. Barcellos (Eds.), Anais do Seminário Internacional sobre Deficiências Minerais em Ruminantes. Porto Alegre: Editora da UFRGS. Recuperado em 12 de dezembro de 2016, http://www.fcav. unesp.br/Home/departamentos/clinicacv/AULUSCAVALIERICARCIOFI/hipocalcemia-vaca-leiteira.pdf

Cseh, S. (1983). Hipomagnesemia. Revisión Bibliográfica. Revista Argentina de Producción Animal, 3(4), 310-314.

Edwards, G., Foster, A., \& Livesey, C. (2009). Use of ocular fluids to aid postmortem diagnosis in cattle and sheep. In Practice, 31(1), 22-25. http://dx.doi.org/10.1136/inpract.31.1.22.

Estima-Silva, P., Molarinho, K. R., Marcolongo-Pereira, C., Soares, M. P., Sallis, E. S. V., Ladeira, S. R. L., \& Schild, A. L. (2016). Morte súbita em bovinos no Sul do Rio Grande do Sul: epidemiologia e diagnóstico. Pesquisa Veterinária Brasileira, 36(1), 19-23. http://dx.doi.org/10.1590/S0100-736X2016000100003.

Goff, J. P. (1998). Ruminant hypomagnesemic tetanies. In J. L. Howard (Ed.), Current veterinary therapy: food animal practice (4th ed.). Philadelphia: W.B. Saunders.

Grabherr, A. C., Widmer, C. A., Iglesias, K. B., Sporkert, A. F., Augsburger, A. M., Mangin, P. A., \& Palmiere, A. C. (2012). Postmortem biochemistry performed on vitreous humor after postmortem CT-angiography. Legal Medicine, 14(6), 297-303. http://dx.doi.org/10.1016/j.legalmed.2012.04.010. PMid:22703847.

Han, S. Q., Qin, Z. Q., Deng, K. F., Zhang, J. H., Liu, N. G., Zou, D. H., Li, Z. D., Shao, Y., Huang, P., \& Chen, Y. J. (2015). Research advances in postmortem chemistry. Fa Yi Xue Za Zhi, 31(4), 287-292. PMid:26665883.

Hanna, P. E., Bellamy, J. E. C., \& Donald, A. (1990). Postmortem eye fluid analysis in dogs, cats, and cattle as an estimate of postmortem serum chemistry profiles. Canadian Journal of Veterinary Research, 54(4), 487-494. PMid:2249181.

Kalra, J., Mulla, A., \& Kopargaonkar, A. (2016). Diagnostic value of vitreous humor in postmortem analysis. SM Journal of Clinical Pathology, 1(1), 1005.

Kaneko, J. J., Harvey, J. W., \& Bruss, M. L. (1997). Clinical biochemistry of domestic animals (5th ed., 932 p. New York: Academic Press.

Lincoln, S. D., \& Lane, V. M. (1985). Concentração de magnésio pós mortem no humor vítreo bovino: Comparação com magnésio de soro antemortem. American Journal of Veterinary Research, 46(1), 160-162. PMid:3970421.

Littledike, E. T., Young, J. W., \& Beitz, D. C. (1981). Common metabolic diseases of cattle: ketosis, milk fever, grass tetany, and downer cow complex. Journal of Dairy Science, 64(6), 1465-1482. http://dx.doi.org/10.3168/jds. S0022-0302(81)82715-4. PMid:7024349.

Mattioli, G. A., Tittarelli, C. M., Giuliodori, M. J., \& Costa, E. F. (2000). Valor diagnóstico del magnesio en humor vítreo en cinco establecimientos con brotes de tetania hipomagnesémica. Analecta Veterinary Journal, 20(2), 39-41.

McCoy, M. A. (2004). Hypomagnesaemia and new data on vitreous humour magnesium concentration as a post-mortem marker in ruminants. Magnesium Research, 17(2), 137-145. PMid:15319147.

McCoy, M. A., \& Kennedy, D. G. (1994). Evaluation of postmortem magnesium concentration in bovine eye fluids as a diagnostic aid for hypomagnesaemic tetany. The Veterinary Record, 135(8), 188-189. http://dx.doi. org/10.1136/vr.135.8.188. PMid:7992479.

McCoy, M. A., Bingham, V., Hudson, A. J., Cantley, L., Hutchinson, T., Davison, G., Fitzpatrick, D. A., \& Kennedy, D. G. (2001). Marcadores bioquímicos pós-mortem de tetania hipomagnesêmica induzida experimentalmente em ovinos. The Veterinary Record, 148(8), 233-237. http://dx.doi.org/10.1136/vr.148.8.233. PMid:11289550.

Mcdowell, L. R. (1992). Minerals in animal and human nutrition. New York: Academic Press.

McLaughlin, P. S., \& McLaughlin, B. G. (1987). Análise química do humor vítreo bovino e porcino: correlação de valores normais com valores químicos séricos e alterações com tempo e temperatura. American Journal of Veterinary Research, 48(3), 467-473. PMid:3565904.

Mendonça Júnior, A. F., Braga, A. P., Rodrigues, A. P. M. S., Sales, L. E. M., \& Mesquita H. C. (2011). Minerais: importância de uso na dieta de ruminantes. ACSA. Agropecuária Científica no Semiarido, 7(1):1-13. 
Metushi, I. G., Fitzgerald, R. L., \& Mc Intyre, I. M. (2016). Assessment and comparison of vitreous humor as an alternative matrix for forensic toxicology screening by GC-MS. Journal of Analytical Toxicology, 40(4), 243247. http://dx.doi.org/10.1093/jat/bkw009. PMid:26945835.

Ramírez, C., Tittarelli, C., Mattioli, G., \& Lasta, G. (1998). Empleo del humor vítreo para la estimación postmortem de la magnesemia en bovinos. Archivos de Medicina Veterinaria, 30(1), 157-160. http://dx.doi.org/10.4067/ s0301-732X1998000100019.

Tormey, W. P. (2016). The potential diagnostic value of vitreous humor analyses at autopsy is not appreciated. Irish Journal of Medical Science, 185(4), 931-934. http://dx.doi.org/10.1007/s11845-016-1449-z. PMid:27037566.

Weiss, W. P. (2004). Macromineral digestion by lactating dairy cows: factors affecting digestibility of magnesium. Journal of Dairy Science, 87(7), 2167-2171. http://dx.doi.org/10.3168/jds.S0022-0302(04)70036-3. PMid:15328230.

Wittwer F., Bohmwald H., Contreras P.A., \& Filoza J. (1987). Análisis de los resultados de perfiles metabólicos en rebaños lechero en Chile. Archivos de Medicina Veterinaria, 9(2):35-45.

Wittwer, F., Urcullú, F., Contreras, P. A., \& Bohmwald, H. (1992). Concentraciones postmortem de minerales, urea y creatinina en humor acuoso y vítreo en vacas como indicadores de sus concentraciones sanguíneas premortem. Archivos de Medicina Veterinaria, 24(1), 61-68.

Zelal, A. (2017). Hypomagnesemia tetany in cattle. Advances in Dairy Research, 5(2):2-9. http://dx.doi. org/10.4172/2329-888X.1000178. 